\title{
Yellow nail syndrome as a cause of unexplained edema
}

\author{
C. Cimini, R. Giunta, R. Utili, E. Durante-Mangoni
}

ABSTRACT: Yellow nail syndrome as a cause of unexplained edema. C. Cimini, R. Giunta, R. Utili, E. Durante-Mangoni.

Yellow nail syndrome is a rare cause of edema due to a disordered lymphatic drainage. We recently observed two cases of long-standing, chronic edema, whose nature could not be understood despite innumerable diagnostic procedures. The diagnosis was suspected based on an attentive clinical exam and confirmed by radionuclide lymph scan.

Yellow nail syndrome has to be considered in the differential diagnosis in cases of systemic edema, as well as long standing pleural effusions, particularly in patients with bronchiectasis or sinusitis. Clues to diagnosis are the presence of dystrophic, yellowish nails, peripheral lymphedema and relapsing pleural effusions and/or ascites.

Long-term control of symptoms is difficult to achieve and may benefit from the judicious use of diuretics and intravenous albumin and by topical alpha-tocopherol. Pleurodesis may be needed. Other pathologic conditions are often associated to yellow nail syndrome and should be ruled out.

Monaldi Arch Chest Dis 2009; 71: 4, 176-179.

Keywords: Edema, Pleural effusion, Differential diagnosis, Onicopathy, Lymphedema.

Institute of Internal Medicine, Second University of Naples Medical School, Naples, Italy.

Correspondence: Dr. Emanuele Durante-Mangoni Cattedra di Medicina Interna - S.U.N., Ospedale Monaldi, Via Bianchi, 80138 Napoli,Italy; e-mail: emanuele.durante@unina2.it

\section{Introduction}

Edema is a cardinal manifestation of disease brought about by a number of different clinical conditions. By definition, it denotes the swelling of soft tissues due to expansion of the interstitial fluid volume.

According to the biochemical features of the fluid, edema is classified as either protein-poor, whose pathogenetic mechanisms are colloid osmotic, hydrostatic or mechanical, or protein-rich, whose major pathogenesis is inflammatory. The fluid appears as a transudate in the former instance, an exudate in the latter, but exceptions may occur.

Clinically, edema can be local or systemic. Local edema is usually due to venous or lymphatic vessel diseases while systemic forms characterise heart failure, decompensated cirrhosis, renal insufficiency or severe protein discrasias.

The excess of fluid in the extracellular space may affect the normal function at cell, tissue and organ level, but most importantly edema implies an underlying disease process.

In clinical practice, it may happen that all major aetiologies of edema are excluded in an individual patient yet its cause remains unclear. In this report, we present our clinical experience with two cases of yellow nail syndrome (YNS), a rare cause of generalised edema of apparently 'obscure' origin.

\section{Case Reports}

Case \#1:

A 71 year old man was referred to hospital because of worsening dyspnoea and severe edema, primarily at lower extremities. His clinical history began five years before admission when he presented bilateral, persistent lower limb edema that was partly responsive to diuretic therapy. For two years prior to admission, he had suffered recurrent episodes of respiratory failure associated with bilateral pleural effusions. Symptoms invariably relapsed a few weeks after pleural fluid taps. The patient was therefore referred to our hospital.

He had never travelled outside Italy and had no history of liver or kidney disease, diabetes mellitus, hypertension or ischemic heart disease. He had never undergone surgery and had no history of lymphangitis or tuberculosis. Physical examination was remarkable for non-pitting edema of the upper and lower limbs, mild ascites and scrotal swelling. He also had dullness and decreased breath sounds at both lung bases.

Laboratory tests were all within normal ranges, including biochemistry, immunology, microbiology and tumour markers. Chest CT scan showed only bilateral pleural effusions. No abnormalities were found at echocardiography. Bronchoscopy was unremarkable and bronchial aspirate cultures were negative. No atypical or neoplastic 
cells were found. Doppler ultrasound of the lower limbs showed normal venous flow and no signs of venous insufficiency or obstruction. Abdominal ultrasound was unremarkable except for bilateral hydrocele.

The case was discussed with the chest surgeons and the patient underwent video-assisted thoracoscopy. About $2500 \mathrm{~mL}$ of chylous fluid was drained and multiple pleural biopsies were performed. The fluid showed high albumin, lactate dehydrogenase and triglyceride levels and few lymphocytes. Pleural histology showed sclero-ialine degeneration but no neoplastic cells. The common causes of a pleural transudate (heart failure, liver cirrhosis, nephropathy, myxo-edema or hypoproteinemia) or pleuritis (lymphoma, metastatic disease, connective tissue disease, infection) were therefore excluded.

At further daily clinical examinations, we noticed the presence of brown-yellowish, thickened nails, showing marked curving and transverse ridging. The lunulae were absent, and there was a distinct hump on all nails (figure 1). YNS was then suspected.

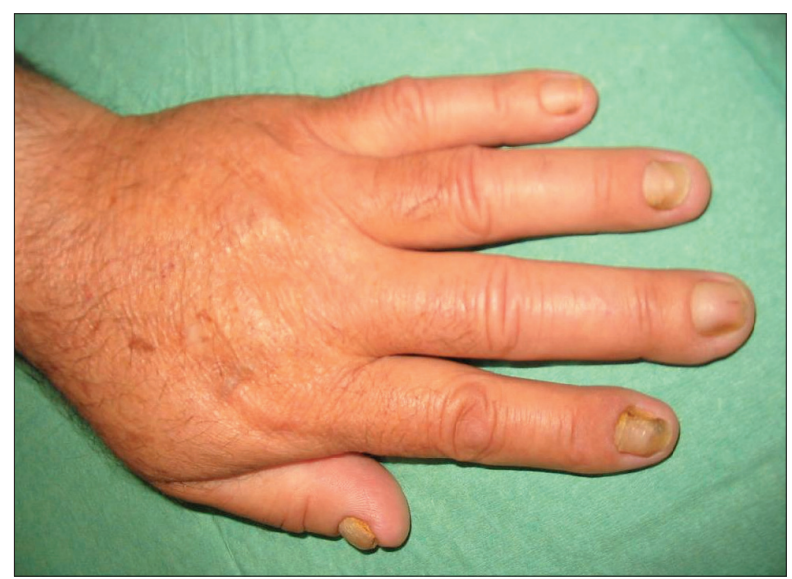

Fig. 1. - The major clinical feature of Yellow Nail Syndrome: typical nail changes in Case \#1.

To evaluate a possible lymphatic drainage deficit, we performed whole body radionuclide lymph scan that displayed loss of lymphatic discharge at the upper and lower limbs in early and late phases with no display of the thoracic duct. These features allowed us to confirm the diagnosis of YNS.

\section{Case \#2:}

A 66 year old man was referred for evaluation of possible cor pulmonale. His clinical history was significant for hypertension for the past 15 years.

He reported that he had suffered with edema of the lower limbs and of the hands for the past 11 years. Six years before admission, he was hospitalised elsewhere and subjected to innumerable clinical, laboratory and imaging studies, leading to a mock diagnosis of 'hypertensive vascular polysclerosis'. A course of treatment with furosemide and intravenous albumin and a low-salt diet yield- ed resolution of lower limb edema. A few months later, the patient conditions deteriorated again, requiring intensification of the diuretic therapy. Nonetheless, the patient remained short of breath and became anasarcatic.

On admission to our hospital, he presented bilateral pleural effusions (figure 2), moderate ascites and severe non-pitting leg and arm edema involving both hands and feet, as well as head and neck swelling. The remaining systems were normal.

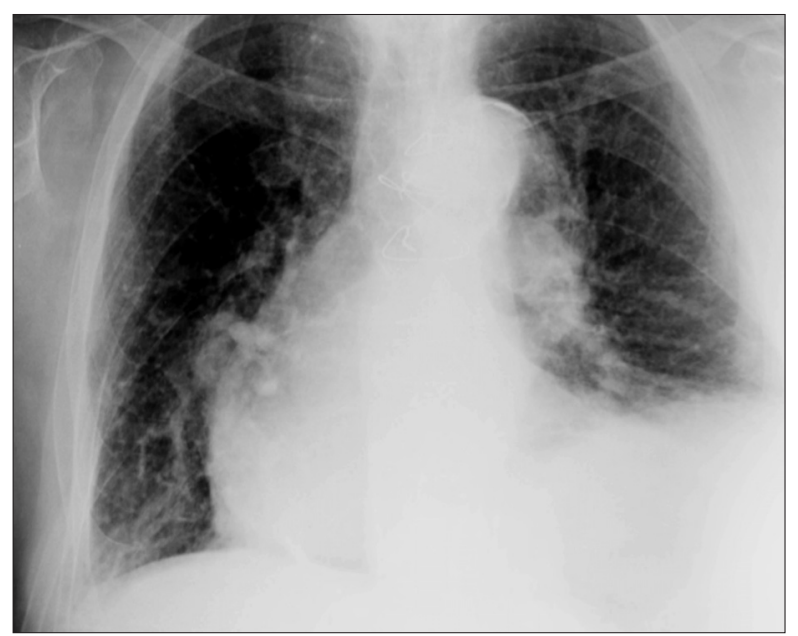

Fig. 2. - Chest X-ray on admission in Case \#2. Note the prominence of the right heart profile.

To evaluate the nature of the pleural effusion, a thoracentesis was performed. The pleural fluid sample was yellowish and dense and showed a high protein content $(4.0 \mathrm{~g} / \mathrm{dL})$ and normal lipid levels. Neither Gram's staining nor culture revealed microrganisms. Mycobacterium tuberculosis polymerase chain reaction assay was also negative. Cytology did not find malignant cells.

The patient underwent a complete check up. Abdominal ultrasound showed mild liver enlargement and ascites. A percutaneous liver biopsy revealed vascular congestion without fibrosis. A paracentesis yielded $500 \mathrm{~mL}$ of yellow fluid (protein content $5.0 \mathrm{~g} / \mathrm{dl}$ ) rich in mononuclear white blood cells. Cytology revealed rare isolated reactive mesothelial cells and typical lymphocytes, but no malignant cells. Transthoracic echocardiography showed a preserved function of both ventricles, minimal mitral valve regurgitation and absence of pericardial effusion. A whole body CT scan and a cardiac MR gave no diagnostic clues.

Inspection of the hands and feet eventually suggested the diagnosis: it revealed yellowing of the fingernails and toenails, showing discrete darker areas, overcurling, loss of cuticle and lunula and hyperkeratosis (figure 3). The patient reported his nails had been - since puberty - thick, brown-yellowish and easy to break. They grew very slowly, sometimes detaching from their bed.

To confirm the diagnosis of YNS, the patient underwent whole body radionuclide lymph scan that displayed a complete and ubiquitous lack of lymphatic discharge (figure 4). 


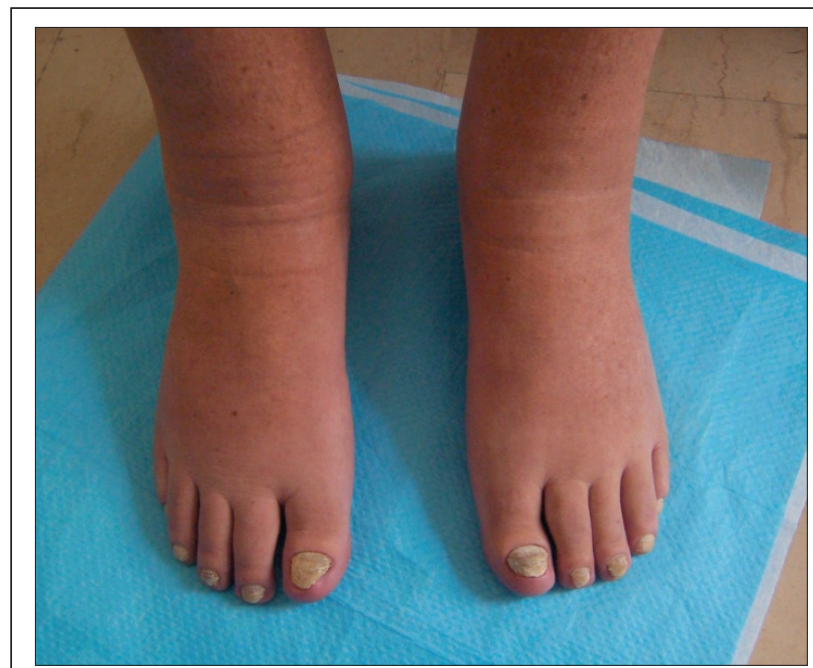

Fig. 3. - Bilateral lower limb edema and nail changes in Case \#2.

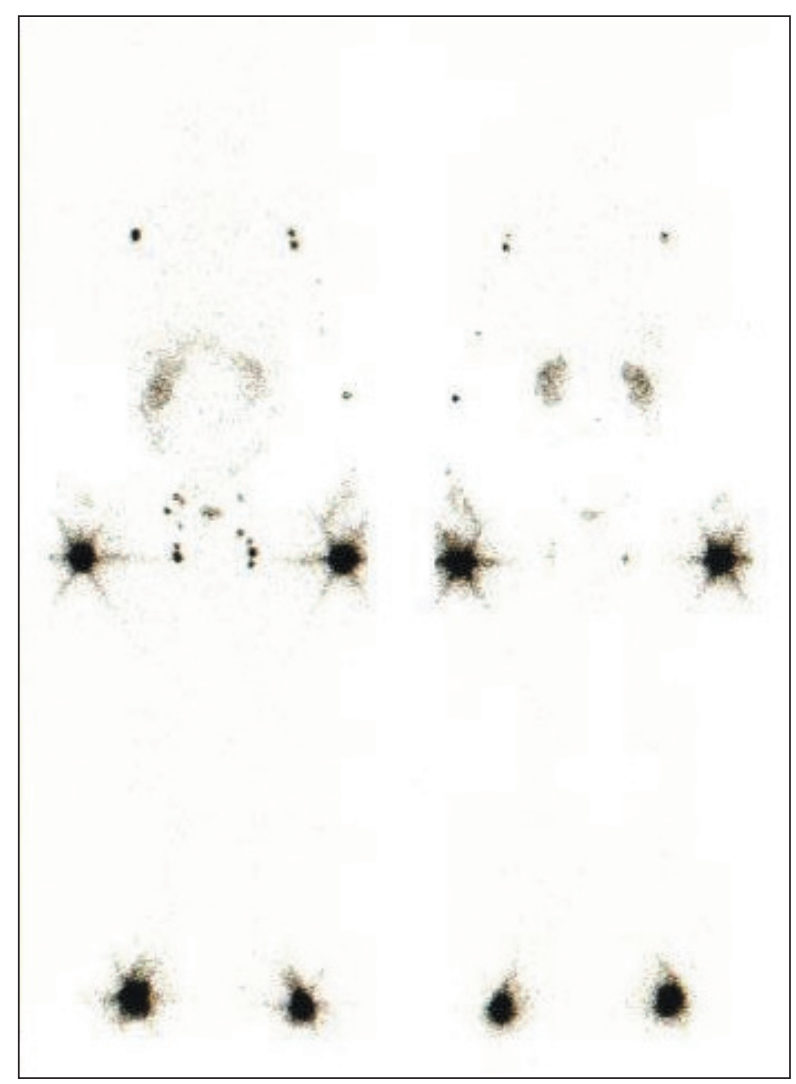

Fig. 4. - Whole-body radionuclide lymph scan showing no display of the peripheral lymphatic tree in Case \#2.

\section{Discussion}

Edema is a major sign of disease. Occasionally, its cause may not be obvious. We recently observed two patients with a long-lasting history of systemic edema and recurrent bouts of decompensation with pleural effusion and ascites. They had gone through a number of diagnostic procedures, but none had provided a clear explanation for the edema. A careful and thoughtful clinical examination was the key to diagnosis of their condition.

YNS is a rare disorder, first described in 1964 [1]. It is characterised by 3 major features: presence of dystrophic, thickened, and slow-growing yellow nails; peripheral lymphedema, most frequently involving lower extremities; and relapsing pleural effusions [2]. However, these three manifestations are simultaneously present in only $27 \%$ of cases. Pleural involvement is often accompanied by other features, mainly bronchiectasis and chronic rhinosinusitis due to bronchial hyper-responsiveness [3]. The age at onset is also widely variable, averaging at 40 years. Throughout the natural course of the disease, yellow nails are present in most cases. The vast majority of patients develop lymphedema of varying severity. In several patients, the initial presentation is related to pleural effusion [2, 3].

Most reported cases are idiopathic, although the syndrome has been described in association with malignancies or autoimmune disorders $[2,5,6]$. Both sporadic cases and familial clusterings of YNS have been reported [2,4-6]. The genetic transmission of YNS is currently being investigated although it seems to have an autosomal dominant inheritance. One causative genetic defect maps at locus $16 \mathrm{q} 24.3$ and affects the forkhead family transcription factor gene MFH1 (FOXC2) [7].

The pathophysiology of YNS is unclear [2, 8]. Lymphatic vessel alterations play an important role. Defective lymph drainage and lymphedema is attributed to hypoplasia of the lymphatic tree. This defect can be demonstrated by peripheral lymph scan, as occurred in our experience. Thus, in YNS edema results from an imbalance between capillary filtration and lymph flow, but an inflammatory component may not been excluded. Indeed, our patients' pleural fluid displayed some characters that are typical of exudative effusions.

There are no approved treatments for YNS [2, $7,9]$. Topical anti-oxidant alpha-tocopherol (vitamin E) has been used to treat nail changes [10]. Judicious use of combined loop and potassium-sparing diuretics together with limb elastic compression may improve symptoms. Percutaneous drain of effusions may occasionally be needed.

In conclusion, YNS is a rare disorder causing edema and effusions. Its treatment is basically symptom-oriented. A diagnosis of YNS should be considered in patients with pleural effusion of unknown cause, especially when it is bilateral or chylous or it occurs in patients with lymphedema. Moreover, YNS should always be contemplated in the differential diagnosis of long-lasting chronic pleural effusions, particularly in patients with bronchiectasis or chronic sinusitis. A lymph scan is useful to demonstrate the defect although a careful clinical exam including nail inspection remains the real clue to diagnosis.

\section{References}

1. Samman PD. The yellow nail syndrome: dystrophic nails associated with lymphedema. Trans St John's Hosp Dermatol Soc 1964; 50: 132.

2. Maldonado F, Ryu JH. Yellow nail syndrome. Curr Opin Pulm Med 2009; 15: 371-375.

3. Maldonado F, Tazelaar HD, Wang CW, Ryu JH. Yellow nail syndrome: analysis of 41 consecutive patients. Chest 2008; 134: 375-381. 
4. Razi E. Familial yellow nail syndrome. Dermatol Online J 2006; 12: 15.

5. Hoque SR, Mansour S, Mortimer PS. Yellow nail syndrome: not a genetic disorder? Eleven new cases and a review of the literature. Brit J Dermatol 2007; 156: 1230-1234.

6. Biscetti F, Straface G, De Angelis G, Flex A. Yellow nails, ankle edema, and pleural effusion. Cleve Clin J Med 2009; 76: 317-8.

7. Finegold DN, Kimak MA, Lawrence EC, et al. Truncating mutations in FOXC2 cause multiple lymphede- ma syndromes. Hum Molec Genet 2001; 10: 11851189.

8. Christu AK, Pastaka C, Papadopoulos D, Klimi E, Gourgoulianis KI. Yellow nail syndrome or diffuse lymphatic network disease. Acta Medica 2002; 45: 181-2.

9. Elmariah SB, Ubriani RR, Kovich O. Yellow nail syndrome. Dermatology Online Journal 2008; 14: 17.

10. Lambert EM, Dziura J, Kauls L, Mercurio M, Antaya RJ. Yellow nail syndrome in three siblings: a randomized double-blind trial of topical vitamin E. Pediatr Dermatol 2006; 23: 390-5.

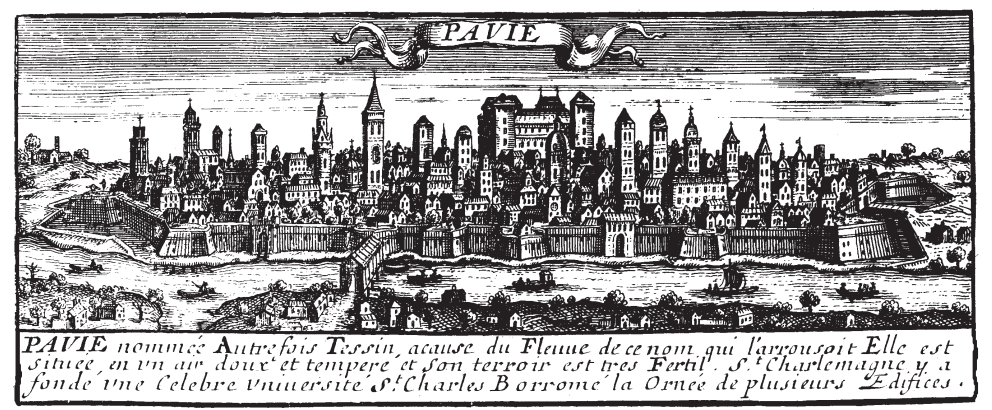

